\title{
Impact of Body Mass Index on COVID-19-Related In- Hospital Outcomes and Mortality
}

\author{
Waqas Ullaha, d, Sohaib Roomi ${ }^{\mathrm{a}}$, Nayab Nadeem ${ }^{\mathrm{a}}$, Rehan Saeed ${ }^{\mathrm{b}}$, Shafaq Tariq ${ }^{\mathrm{a}}$, Moataz Ellithi ${ }^{\mathrm{b}}$, \\ Shujaul Haq a , Ahmad Arslana, John Madara a, Margot Boigon ${ }^{a}$, \\ Donald C. Haas ${ }^{\mathrm{a}}$, David L. Fischman ${ }^{\mathrm{c}}$
}

\begin{abstract}
Background: Given the high prevalence of obesity around the globe, patients with coronavirus disease 2019 (COVID-19) are at an increased risk of devastating complications.

Methods: A retrospective cohort study was performed to determine the association of basal metabolic index (body mass index (BMI)) with the need for invasive mechanical ventilation (IMV), dialysis, upgrade to an intensive care unit (ICU) and mortality. Independent $t$-test and multivariate logistic regression analysis were performed to calculate mean differences and adjusted odds ratios (aORs) with its $95 \%$ confidence interval $(\mathrm{CI})$, respectively.

Results: A total of 176 consecutive patients with confirmed COVID-19 diagnosis were included. The mean age was 62.2 years, with $51 \%$ being male patients. The mean BMI for non-surviving patients was significantly higher compared to patients surviving on the seventh day of hospitalization ( $35 \mathrm{vs} .30 \mathrm{~kg} / \mathrm{m}^{2}, \mathrm{P}=0.022$ ). Similarly, patients requiring IMV had a higher BMI (33 vs. $29, \mathrm{P}=0.002$ ) compared to nonintubated patients. The unadjusted OR for patients with a higher BMI requiring IMV ( $56 \%$ vs. $28 \%$, OR: $3.3,95 \% \mathrm{CI}: 1.6-7.0, \mathrm{P}=0.002)$ and upgrade to ICU (46\% vs. $28 \%$, OR; $2.2,1.07$ - 4.6, P = 0.04) were significantly higher compared to patients with a lower BMI. Similarly, patients with a higher BMI had higher in-hospital mortality (21\% vs. 9\%, OR: $3.2,95 \%$ CI: $1.3-8.2, \mathrm{P}=0.01$ ) compared to patients with a normal BMI. Despite a numerical advantage in the lower BMI group, there was no significant difference between the two groups in terms of the need for dialysis ( $5 \%$ vs. $13 \%$, OR: $3.8,13 \%$ vs. $4 \%, 1.1-14.1$, P $=0.07$ ). aORs controlled for baseline comorbidities and medications mirrored the overall results, except for the need to upgrade to ICU.
\end{abstract}

Conclusions: In patients with confirmed COVID-19, morbid obesity

Manuscript submitted May 28, 2020, accepted February 3, 2021

Published online April 27, 2021

\footnotetext{
aA Abington Jefferson Health, Abington, PA, USA

bUniversity of South Dakota, Vermillion, SD, USA

'Thomas Jefferson University, Philadelphia, PA, USA

${ }^{\mathrm{d}}$ Corresponding Author: Waqas Ullah, Department of Internal Medicine, Abington Jefferson Health, Abington, PA, USA.

Email: waqasullah.dr@gmail.com
}

doi: https://doi.org/10.14740/jocmr4239 serves as an independent risk factor of high in-hospital mortality and the need for IMV.

Keywords: Body mass index; COVID-19; In-hospital mortality; Invasive mechanical ventilation

\section{Introduction}

The USA is the current epicenter of the novel coronavirus pandemic with over 1.6 million cases and nearly 100,000 deaths. While the true mortality and morbidity caused by coronavirus disease 2019 (COVID-19) will take years to become apparent, we do know that certain groups of people particularly the elderly and those with certain comorbidities are at a significantly higher risk of worse outcomes. Among these conditions is obesity, with the USA currently leading the developed world in terms of obesity among its citizens (42\%), this puts a large population at measurably higher risk of major complications, delayed recovery and potentially higher mortality $[1,2]$.

An analysis of a large cohort of COVID-19 patients by Lighter et al demonstrated 1.8 and 3.6 times greater probability for admission to critical care units for obese (body mass index (BMI) 30 - 34.9) and morbidly obese patients (BMI > 35), respectively [3]. Similarly, in a study by Kalligeros et al and Petrilli et al, BMI $\geq 35 \mathrm{~kg} / \mathrm{m}^{2}$ was associated with a significantly higher rate of admission to intensive care unit (ICU) and need for invasive mechanical ventilation (IMV) $[4,5]$.

With a high burden of COVID19 and limited healthcare resources, it is imperative to determine the impact of obesity not only on in-hospital complications but also on mortality, to better inform clinical decision making and resource allocation.

\section{Materials and Methods}

\section{Study design and participants}

This retrospective cohort study included consecutive adult inpatients ( $\geq 18$ years old) from Abington Hospital, Jefferson Health, Pennsylvania, USA. All patients had a confirmed diagnosis of COVID-19 between March 1, 2020, and May 10, 
2020. The study was approved by the Institutional Review Board (IRB) and the requirement for informed consent was waived by the Research Ethics Committee (REC). All procedures described in the study have been actuated according to ethical principles for medical research involving human subject stated in the Declaration of Helsinki.

\section{Data collection}

Clinical, demographic, laboratory, treatment, and outcome data were extracted from electronic medical records (Sunrise) using a standardized data collection form. All authors contributed to data retrieval and an independent author adjudicated any difference in interpretation between the data extractors. Severe acute respiratory syndrome coronavirus 2 (SARS-CoV-2) detection in respiratory specimens (throat swabs) was done by next-generation sequencing or real-time qualitative polymerase chain reaction (RT-qPCR) methods. The laboratory values, cut-off variables and methods for laboratory confirmation of SARS-CoV-2 infection were standardized. Data regarding baseline comorbidities included a history of diabetes mellitus (DM), hypertension (HTN), chronic kidney disease (CKD) and coronary artery disease (CAD). In hospital medications used included hydroxychloroquine (HCQ), tocilizumab, steroids and anticoagulation (AC). Routine blood work included coagulation profile, complete blood count, serum biochemical tests (renal function, C-reactive protein (CRP), D-dimer, lactate dehydrogenase (LDH), myocardial enzymes (troponin T (TnT)) and serum ferritin. Chest radiographs or computed tomography (CT) scans were also done for most inpatients where clinically indicated. The criteria for discharge were absence of fever, freedom from symptoms, and substantial clinical or radiological improvement for at least 1 day.

Based on the standard definition of the World Health Organization (WHO), patients were divided into two groups, severely obese population with a BMI $>35 \mathrm{~kg} / \mathrm{m}^{2}$ and those who were not severely obese (BMI $\left.<34.9 \mathrm{~kg} / \mathrm{m}^{2}\right)$. Patients with BMI $18.5-24.9 \mathrm{~kg} / \mathrm{m}^{2}$ were classified as having a normal BMI, and those with BMI greater than $40 \mathrm{~kg} / \mathrm{m}^{2}$ were noted as very severely obese.

\section{Statistical analysis}

Continuous variables were presented as mean and standard deviation (SD); categorical variables were reported in percentages and proportions. A Chi-square $\left(\chi^{2}\right)$ test was used for comparison of categorical data. Fisher exact test was only adopted if the expected count in more than $20 \%$ cells was less than 5. To quantify the association between the dichotomous categorical variables, an unadjusted odds ratio (OR) was obtained using the Cochran-Mantel-Haenszel method. To explore the risk factors and gauge the impact of potential effect modifiers (covariates) on our endpoints (in-hospital death, need for an upgrade, ventilators and dialysis) binomial and multinomial logistic regression models were applied as appropriate. The differences in the baseline comorbidities (DM, HTN, CAD,
CKD) and medication use (HCQ, tocilizumab, AC and steroids) were accounted for to obtain an adjusted OR (aOR) for all outcomes. The Hosmer-Lemeshow (HL) goodness-of-fit test was used to predict the fitness of logistic regression models for applicability to categorical data. The mean BMI values for baseline comorbidities, in-hospital complications and clinical endpoints were also compared for both comparison groups. For normally and abnormally distributed continuous data, an independent sample $t$-test and Mann-Whitney U test were utilized, respectively. A one-way analysis of variance (ANOVA) was used to compare differences in the mean of continuous variables for multiple in-hospital complications. A two-sided $\alpha$ of less than 0.05 was considered statistically significant corroborating inference from a $95 \%$ confidence interval (CI). Statistical analyses were performed using the SPSS software (version 25).

\section{Results}

\section{Demographics and baseline characteristics}

A total of 176 consecutive patients (137 with BMI $<34.9$ and 39 with $\mathrm{BMI}>35)$ were included. The mean age for lower BMI vs. higher BMI groups was 64.8 vs. $62.6(\mathrm{P}=0.02)$, respectively. The baseline comorbidities across all groups were comparable except that the lower BMI group had a higher percentage of chronic obstructive pulmonary disease (COPD) (P $=0.01)$ and DM (0.046). The proportion of other comorbidities and medication use (HCQ, tocilizumab, AC, steroids) were comparable across both groups $(\mathrm{P} \geq 0.05)$ (Fig. 1 and Table 1).

\section{Mean differences in BMI across outcomes}

The mean BMI for patients who were alive $(153 / 176)$ at the 7-day of hospitalization was $30.2 \pm 8.9$ compared to a BMI of $35.4 \pm 13.8$ for patients who died $(23 / 176)$. The mean difference of BMI was -5.15 (95\% CI: -9.5 to -7.4$)$, significantly lower in patients who were alive $(\mathrm{P}=0.022)$. Similarly, $60 / 176$ patients who were intubated had a higher BMI of $33.9 \pm 11.2$ compared to non-intubated patients $(\mathrm{n}=116 / 176$, BMI: $29.2 \pm$ 8.4). The mean difference in the BMI was significantly lower in the non-ventilated group $(-4.7(-7.7$ to -1.7$) \mathrm{P}=0.002)$. There was no significant difference in the BMI of patients receiving $\mathrm{HCQ}$ vs. no HCQ $(\mathrm{P}=0.21)$, tocilizumab vs. no tocilizumab $(\mathrm{P}=0.44)$, or AC $(\mathrm{P}=0.14)$. Similarly, a higher BMI was not associated with an increased risk of new-onset kidney failure requiring dialysis $(\mathrm{P}=0.29)$ or an upgrade to the critical care unit $(\mathrm{P}=0.14)$ (Fig. 2, Table 2).

The incidence of in-hospital COVID-19 related complications was rare and not significantly impacted by $\mathrm{BMI}(\mathrm{P}=$ 0.65). The mean BMI for patients developing deep vein thrombosis (DVT), pulmonary embolism (PE), sepsis, acute kidney injury (AKI), atrial fibrillation (AF), junctional rhythm and bleeding was $24.50 \pm 2.12 \mathrm{~kg} / \mathrm{m}^{2}, 30.33 \pm 5.51 \mathrm{~kg} / \mathrm{m}^{2}, 27.89$ $\pm 14.87 \mathrm{~kg} / \mathrm{m}^{2}, 38.27 \pm 17.70 \mathrm{~kg} / \mathrm{m}^{2}, 31.50 \pm 6.32 \mathrm{~kg} / \mathrm{m}^{2}$ and $21.0 \mathrm{~kg} / \mathrm{m}^{2}$, respectively. Intriguingly, the BMI for the patient developing cardiac arrest and AF was $52 \mathrm{~kg} / \mathrm{m}^{2}$ (Supplementa- 


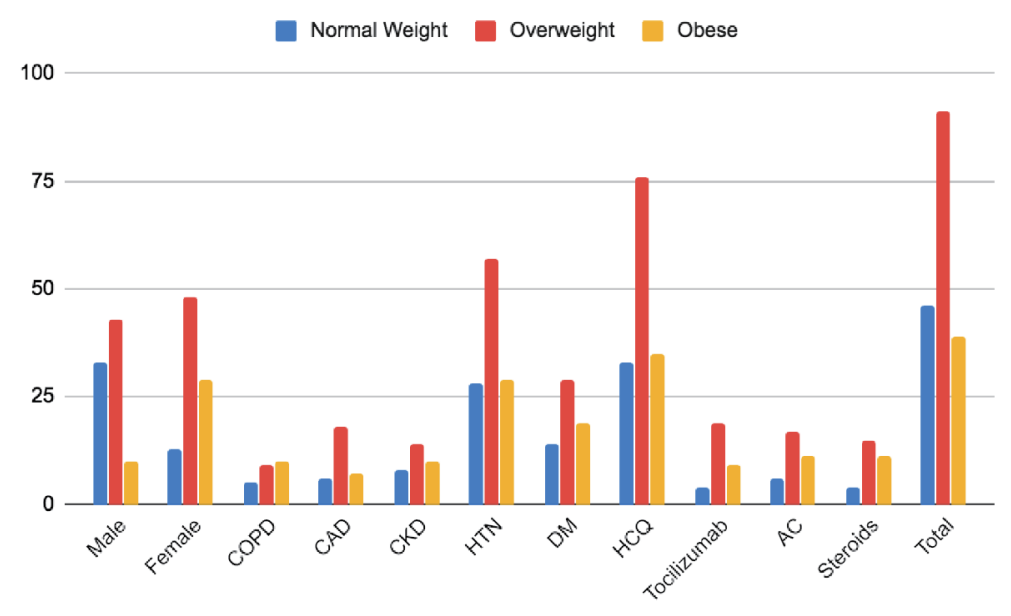

Figure 1. Baseline characteristics of patients in different groups. CAD: coronary artery disease; COPD: chronic obstructive pulmonary disease; CKD: chronic kidney disease; HTN: hypertension; DM: diabetes mellitus; HCQ: hydroxychloroquine; AC: anticoagulation.

Table 1. Baseline Characteristics of the Included Population Across Comparison Groups

\begin{tabular}{llll} 
& BMI $<34.9$ & BMI $>35$ & P value \\
Male & $76(88.4 \%)$ & $10(11.6 \%)$ & $0.001^{*}$ \\
Female & $61(67.8 \%)$ & $29(32.2 \%)$ & 0.95 \\
CAD & $24(77.4 \%)$ & $7(22.6 \%)$ & $0.013^{*}$ \\
COPD & $14(58.3 \%)$ & $10(41.7 \%)$ & 0.171 \\
CKD & $22(68.8 \%)$ & $10(31.3 \%)$ & 0.155 \\
HTN & $85(74.6 \%)$ & $29(25.4 \%)$ & $0.046^{*}$ \\
DM & $43(69.4 \%)$ & $19(30.6 \%)$ & 0.369 \\
Tocilizumab & $23(71.9 \%)$ & $9(28.1 \%)$ & 0.146 \\
HCQ & $109(75.7 \%)$ & $35(24.3 \%)$ & $0.036^{*}$ \\
Steroids & $19(63.3 \%)$ & $11(36.7 \%)$ & 0.111 \\
AC & $23(67.6 \%)$ & $11(32.4 \%)$ & \\
\hline
\end{tabular}

${ }^{*} \mathrm{P}<0.05 . \mathrm{BMI}$ : body mass index; CAD: coronary artery disease; COPD: chronic obstructive pulmonary disease; CKD: chronic kidney disease; HTN: hypertension; DM: diabetes mellitus; HCQ: hydroxychloroquine; AC: anticoagulation.

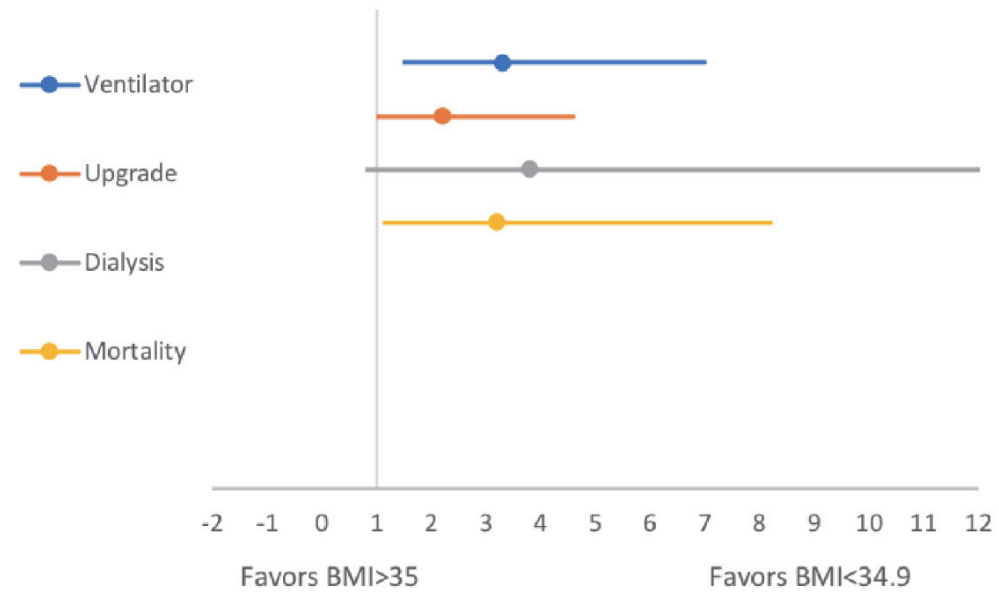

Figure 2. Forest plots comparing in-hospital endpoints across high and lower BMI groups. BMI: body mass index. 
Table 2. Mean BMI Values Across Different Outcomes and Medication Groups

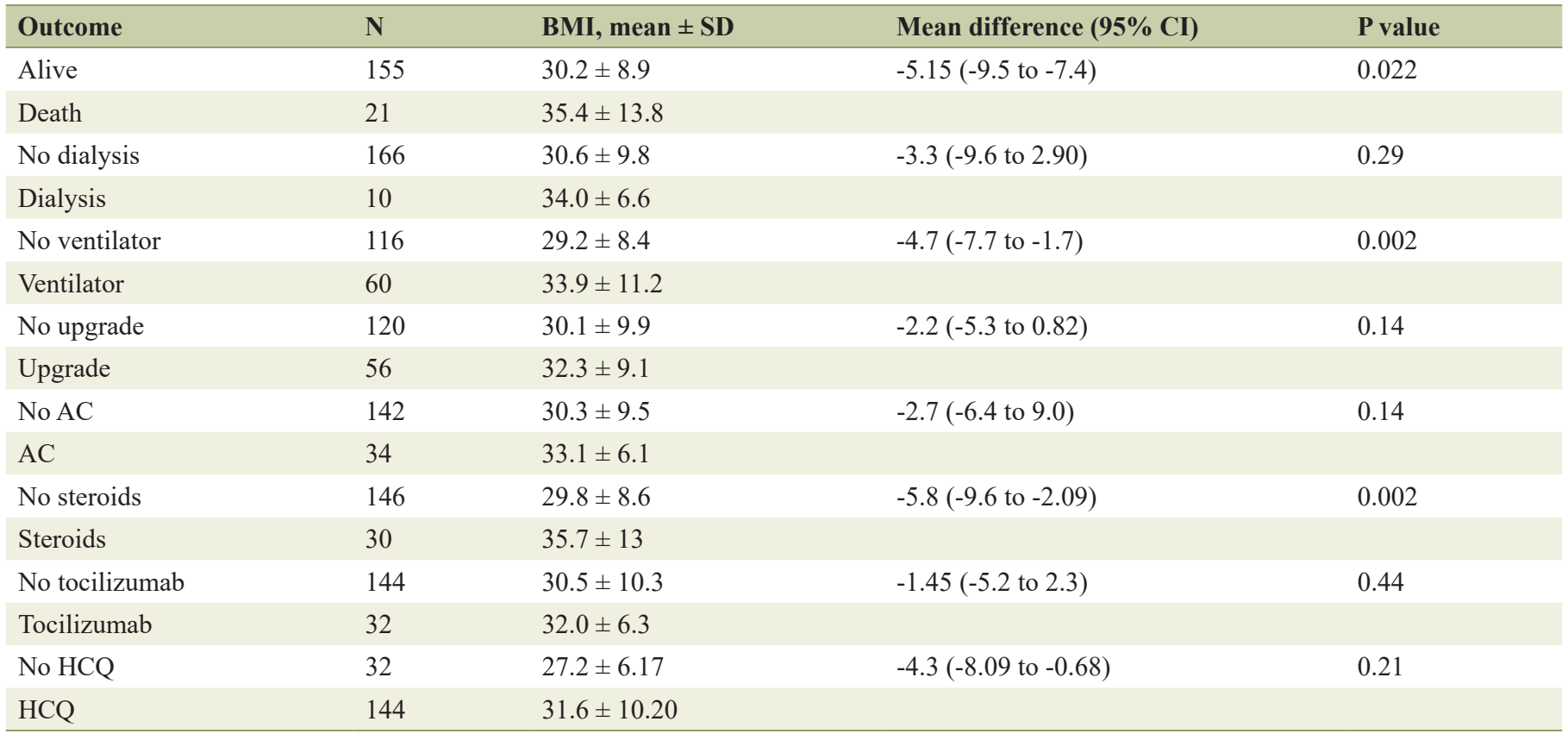

SD: standard deviations; Cl: confidence interval; BMI: body mass index; HCQ: hydroxychloroquine; AC: anticoagulation.

ry Material 1, www.jocmr.org). Similarly, there was no significant difference in the mean values of inflammatory markers and laboratory investigations of patients with higher and lower BMI (Supplementary Material 2, www.jocmr.org).

\section{ORs of outcomes}

The unadjusted OR for patients with a higher BMI requiring IMV (56\% vs. $28 \%$, OR: $3.3,95 \%$ CI: $1.6-7.0, \mathrm{P}=0.002)$ and upgrade to ICU (46\% vs. $28 \%$, OR: $2.2,1.07-4.6, \mathrm{P}=$ $0.04)$ were significantly higher compared to patients with a lower BMI. Similarly, patients with a higher BMI had higher in-hospital mortality (21\% vs. 9\%, OR: $3.2,95 \%$ CI: $1.3-8.2$,
$\mathrm{P}=0.01$ ) compared to patients with a normal BMI. Despite a numerical advantage in the lower BMI group, there was no significant difference between the two groups in terms of the need for dialysis (5\% vs. 13\%, OR: 3.8, 13\% vs. 4\%, 1.1 14.1, $\mathrm{P}=0.07)$.

A multivariate regression model was used to adjust the observed ORs for baseline comorbidities and medications, including DM, HTN, CKD, CAD, use of AC at home, HCQ, tocilizumab, steroids and therapeutic AC during the hospital stay. The aORs mirrored the overall findings of unadjusted ORs with one exception. In contrast to the unadjusted OR, there was no significant difference in the rate of an upgrade to the ICU for patients with high and low BMI groups (aOR: 1.7, 0.7 - 3.9, $\mathrm{P}=0.17$ ) (Fig. 3, Table 3).

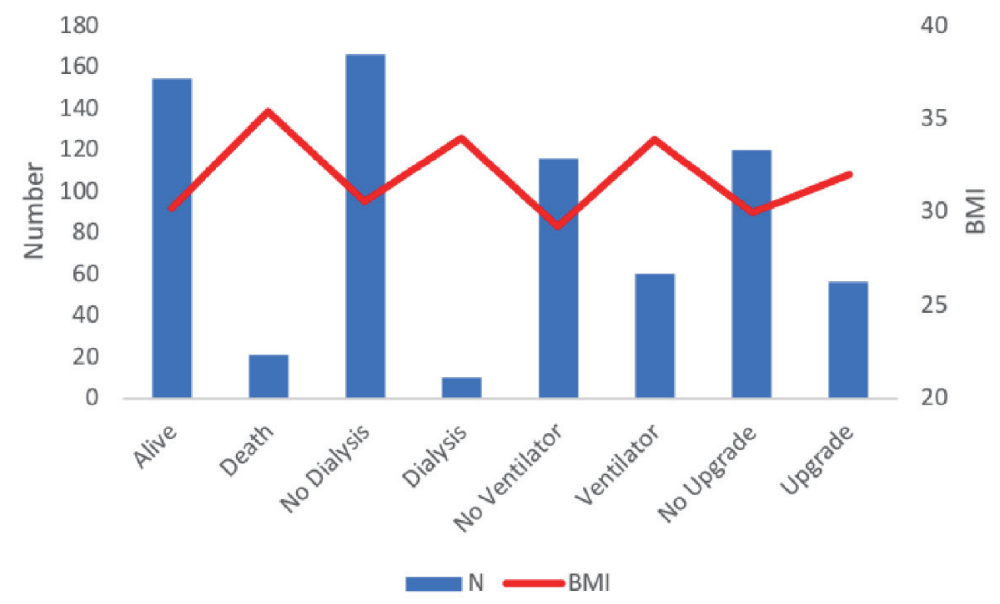

Figure 3. Mean BMI values and number of patients across different in-hospital endpoints. BMI: body mass index. 
Table 3. Unadjusted and Adjusted Odds Ratios of Outcomes in Normal and Obese BMI Groups

\begin{tabular}{|c|c|c|c|c|c|c|c|}
\hline Outcomes & $\mathbf{N}$ & BMI $<34$ & BMI $>35$ & Unadjusted odds & P value & Adjusted odds ratio (aOR) & P value \\
\hline Vent & 60 & $38(28 \%)$ & $22(56 \%)$ & OR $3.3(1.6-7.0)$ & 0.002 & aOR $2.6(1.17-6.1)$ & 0.01 \\
\hline No vent & 116 & $99(72 \%)$ & $17(44 \%)$ & & & & \\
\hline No upgrade & 120 & $99(72 \%)$ & $21(54 \%)$ & & & & \\
\hline Dialysis & 10 & $5(4 \%)$ & $5(13 \%)$ & OR $3.8(1.1-14.1)$ & 0.07 & aOR $3.6(0.8-15.7)$ & 0.08 \\
\hline Alive & 153 & $124(91 \%)$ & $29(79 \%)$ & & & & \\
\hline
\end{tabular}

BMI: body mass index.

\section{Discussion}

Our study reveals that obesity is an independent risk factor for worse outcomes in COVID-19. Patients with a BMI $>35 \mathrm{~kg} /$ $\mathrm{m}^{2}$ have three times higher odds for mortality and respiratory complications necessitating IMV compared to patients with a BMI lower than $34.9 \mathrm{~kg} / \mathrm{m}^{2}$. There were substantial differences in the BMIs of patients who survived COVID-19 compared with those who succumbed to the disease $(5.15, \mathrm{P}=0.02)$. A similar difference was observed in patients who required mechanical ventilation versus those in whom the disease severity did not progress as far $(4.7, \mathrm{P}=0.002)$. Although patients with a higher BMI seemed to have a greater need for higher-level care, this trend could have been driven by multiple comorbidities as evidenced by an identical aOR $(\mathrm{P}=0.14)$. Partly contributing to this was also the higher tendency to opt for "comfort measures" or "no escalation of care" in obese patients, precluding an upgrade to the ICU in patients whose clinical condition otherwise would require it.

Obesity has traditionally been linked to severe respiratory infections. Previous epidemiological and clinical studies have shown that obesity increases the rate of hospitalization as well as death in patients with influenza type A (H1N1) [6-8]. These findings were later validated by Kwong et al and Maccioni et al, who also reported that obese patients were more likely to get hospitalized due to upper and lower respiratory tract complications $[7,8]$. With the recent outbreak, early evidence from China showed a similar association between obesity and COVID-19. Cai et al observed that overweight and obese patients had two-fold higher odds of suffering from severe pneumonia when controlled for potential confounders [9]. In line with these studies, our study demonstrated even higher odds for severe respiratory compromise requiring IMV when adjusted for baseline comorbidities.

It is believed that both mechanical and inflammatory mechanisms contribute to obesity-related adverse outcomes in COVID-19. Obesity results in reduced ventilation by reducing diaphragmatic excursion and limiting chest wall mobility. Additionally, adipose tissue plays a role in immunological response by producing a variety of adipokines and pro-inflammatory cytokines, including leptin, interleukins 4 and 6 , interferon, tumor necrosis factor, adiponectin, resistin, and visfatin $[10,11]$. While our study revealed an increased risk for IMV and mortality, surprisingly, the inflammatory markers including mean $\mathrm{D}$-dimer $(\mathrm{P}=0.99)$, ferritin $(\mathrm{P}=0.81)$ and CRP $(\mathrm{P}=0.31)$ on both day 1 and day 7 of hospitalizations were not impacted by higher BMI. These findings indicate either that the respiratory complications and mortality in obesity in COVID-19 patients could be independent of inflammation or that inflammatory markers lag behind the said complications. It may be that more than inflammation, a higher resistance in their airways, lower lung volumes, and weaker respiratory muscles due to obesity play a major role in respiratory complications in COVID-19.

Apart from the above-mentioned mechanisms, studies have shown that viral replication rates are higher in the cells of patients who are obese compared to those with normal BMI, contributing to a higher susceptibility to viral infection [12, 13]. Studies done to assess the immune response to vaccination have also shown a consistently poorer response in people with obesity [14]. This lends further credence to the theory that adipose tissue driven immunological changes attenuate an effective response to viral infection. A previous analysis of 124 ICU patients with COVID-19 in a French hospital showed a direct correlation of BMI with IMV $(\mathrm{P}<0.01)$, the requirement being highest in those with BMI $>35 \mathrm{~kg} / \mathrm{m}^{2}(85.7 \%)$ [15]. Our findings not only show a similar increase in the need for IMV, but also highlight three-fold higher odds of in-hospital mortality in patients with high BMI. The association is independent of age, comorbidities, or therapeutic strategies employed.

We believe that the collision of the COVID-19 pandemic with the ongoing endemic of obesity poses major clinical challenges for physicians, and has significant logistical implications for the healthcare sector at large. North America and Western Europe are not only the current hubs of COVID-19 but also have the highest prevalence of obesity [1]. Providing intensive care, with its attendant high resource consumption, to these patients represents a challenge for healthcare systems in these regions. The need for more bariatric beds, mechanical ventilators, expertise in intubation and skilled nursing staff (required to position and transport obese patients) rises with each passing day. Severe respiratory complications along with difficulties faced by obese patients during diagnostic imaging further compromise the medical care of such patients. Our study highlights the higher risk of adverse outcomes in this pa- 
tient population, allowing physicians to not only anticipate and prognosticate these unfortunate outcomes but also to inform decisions about resource allocation.

\section{Limitations}

The major limitation of our study was the small sample size from a single institution. Although the retrospective cohort study design used can estimate associations only; similar to a prospective design, our study does have the strength of certainty regarding the temporal sequence of the exposures and outcomes. Likely due to small sample size, CIs were wide and the threshold of statistical significance could not be achieved for multiple comparisons. Although the overall findings were adjusted for covariates, including baseline comorbidities and medications, the impact of unmeasured confounders such as initiation of several complementary therapies at the treating physician's discretion, could not be determined. Moreover, by excluding patients still in the hospital, the case fatality ratio in our study cannot reflect the true mortality of COVID-19. Despite the limited sample size, by adjusting adult patients with the confirmed disease, we believe our population is representative of the real-world cohort.

\section{Conclusions}

In patients with confirmed COVID-19, morbid obesity appears to be an independent risk factor of high in-hospital mortality and the need for IMV.

\section{Supplementary Material}

Suppl 1. Mean BMI values across in-hospital complications. Suppl 2. Differences in the lab findings of patients with different BMI.

\section{Acknowledgments}

None to declare.

\section{Financial Disclosure}

None to declare.

\section{Conflict of Interest}

None to declare.

\section{Informed Consent}

Not applicable.

\section{Author Contributions}

Conceptualization: Waqas Ullah. Data curation and formal analysis: Sohaib Roomi. Investigation and methodology: Rehan Saeed and Nayab Nadeem. Project administration: Margot Boigon and Donald C. Haas. Resources, software, supervision and validation: David L. Fischman and John Madara. Writing (original draft): Shafaq Tariq and Moataz Ellithi. Writing (review and editing): Shujaul Haq and Ahmad Arslan.

\section{Data Availability}

The data supporting the findings of this study are available from the corresponding author upon reasonable request.

\section{References}

1. World Health Organization. Prevalence of obesity among adults, BMI $\geq 30$, age-standardized Estimates by WHO region. Available from: https://apps.who.int/gho/data/ view.main.REGION2480A?lang=en. Accessed May 10, 2020.

2. Centers for Disease Control and Prevention. Overweight \& obesity. Updated February 27, 2020. https://www.cdc. gov/obesity/data/adult.html. Accessed March 18, 2020.

3. Lighter J, Phillips M, Hochman S, Sterling S, Johnson D, Francois F, Stachel A. Obesity in patients younger than 60 years is a risk factor for COVID-19 hospital admission. Clin Infect Dis. 2020;71(15):896-897.

4. Kalligeros M, Shehadeh F, Mylona EK, Benitez G, Beckwith CG, Chan PA, Mylonakis E. Association of obesity with disease severity among patients with coronavirus disease 2019. Obesity (Silver Spring). 2020;28(7):12001204.

5. Petrilli CM, Jones SA, Yang J, Rajagopalan H, O’Donnell L, Chernyak Y, Tobin KA, et al. Factors associated with hospitalization and critical illness among 4,103 patients with COVID-19 disease in New York City. medRxiv. 2020.

6. Morgan OW, Bramley A, Fowlkes A, Freedman DS, Taylor TH, Gargiullo P, Belay B, et al. Morbid obesity as a risk factor for hospitalization and death due to 2009 pandemic influenza A(H1N1) disease. PLoS One. 2010;5(3):e9694.

7. Kwong JC, Campitelli MA, Rosella LC. Obesity and respiratory hospitalizations during influenza seasons in Ontario, Canada: a cohort study. Clin Infect Dis. 2011;53(5):413-421.

8. Maccioni L, Weber S, Elgizouli M, Stoehlker AS, Geist I, Peter HH, Vach W, et al. Obesity and risk of respiratory tract infections: results of an infection-diary based cohort study. BMC Public Health. 2018;18(1):271.

9. Cai Q, Chen F, Wang T, Luo F, Liu X, Wu Q, He Q, et al. Obesity and COVID-19 Severity in a Designated Hospital in Shenzhen, China. Diabetes Care. 2020;43(7):13921398.

10. Trayhurn P, Wood IS. Adipokines: inflammation and 
the pleiotropic role of white adipose tissue. Br J Nutr. 2004;92(3):347-355.

11. Galic S, Oakhill JS, Steinberg GR. Adipose tissue as an endocrine organ. Mol Cell Endocrinol. 2010;316(2):129139.

12. Travanty E, Zhou B, Zhang H, Di YP, Alcorn JF, Wentworth DE, Mason R, et al. Differential susceptibilities of human lung primary cells to H1N1 influenza viruses. J Virol. 2015;89(23):11935-11944.

13. Huang CG, Lee LA, Wu YC, Hsiao MJ, Horng JT, Kuo $\mathrm{RL}$, Huang $\mathrm{CH}$, et al. A pilot study on primary cul- tures of human respiratory tract epithelial cells to predict patients' responses to H7N9 infection. Oncotarget. 2018;9(18):14492-14508.

14. Green WD, Beck MA. Obesity impairs the adaptive immune response to influenza virus. Ann Am Thorac Soc. 2017;14(Supplement_5):S406-S409.

15. Simonnet A, Chetboun M, Poissy J, Raverdy V, Noulette J, Duhamel A, Labreuche J, et al. High prevalence of obesity in severe acute respiratory syndrome coronavirus-2 (SARS-CoV-2) requiring invasive mechanical ventilation. Obesity (Silver Spring). 2020;28(7):1195-1199. 